\title{
Síndrome CLIPPERS con distribución atípica de las lesiones en la resonancia magnética cerebral
}

\author{
Beatrice Canneti, Antonio J. Mosqueira, Francisco Gilo, Teresa Carreras, Antonio Barbosa, \\ Virginia Meca-Lallana, José Vivancos
}

Servicio de Neurología (B. Canneti, A.J. Mosqueira, F. Gilo, T. Carreras, V. Meca-Lallana, J. Vivancos); Servicio de Radiodiagnóstico (A. Barbosa); Hospital Universitario de La Princesa: Instituto de Investigación Sanitaria Princesa. Madrid, España.

Correspondencia: Dra. Beatrice Canneti Heredia. Servicio de Neurología. Hospital Universitario de La Princesa. Diego de León, 62. E-28006 Madrid.

E-mail:

canneti.heredia@gmail.com

Aceptado tras revisión externa: 22.07.13.

Cómo citar este artículo: Canneti B, Mosqueira AJ, Gilo F, Carreras T, Barbosa A, MecaLallana V, et al. Síndrome CLIPPERS con distribución atípica de las lesiones en la resonancia magnética cerebral. Rev Neurol 2013; 57 :

354-8.

(c) 2013 Revista de Neurología

\section{Introducción}

El síndrome CLIPPERS (chronic lymphocytic inflammation with pontine perivascular enhancement responsive to steroids) se caracteriza por un proceso inflamatorio del sistema nervioso central descrito recientemente por Pittock et al [1], cuyo rasgo diagnóstico distintivo es la aparición de lesiones puntiformes en el tronco del encéfalo en la resonancia magnética (RM). En su trabajo se recogen ocho pacientes con características clínicas, radiológicas y anatomopatológicas comunes que incluyen: presencia de síntomas troncoencefálicos episódicos; alteraciones en la RM, consistentes en lesiones puntiformes y curvilíneas que realzan con contraste, localizadas en el tronco del encéfalo (principalmente en el puente); y observación de un infiltrado de células $\mathrm{T}$ en los espacios perivasculares del tronco en la biopsia cerebral. Además, se describe una buena respuesta, con mejoría clínica y radiológica tras el tratamiento con corticoides y la tendencia a la recidiva tras su supresión.

\section{Caso clínico}

Mujer de 40 años con diabetes gestacional como único antecedente de interés que acudió al servicio de urgencias por cuadro de visión doble, de inicio insidioso y curso progresivo, de una semana de evolución. Refería dificultad para articular el habla y cierta inestabilidad al caminar. No había presentado fiebre, artralgias o síntomas en la piel. Tampoco aquejaba síntomas sugestivos de síndrome seco ni de síndrome constitucional. Existía fluctuación de la clínica, sin predominio horario.

En la exploración neurológica realizada en ese momento destacaba una diplopía binocular en la mirada horizontal-superior, de predominio en la mirada extrema hacia la derecha y al enfocar a lo lejos, sin que se objetivasen restricciones de la musculatura extrínseca ocular, aunque sí cierta dificultad en el seguimiento. Se observó un nistagmo horizontorrotatorio en la mirada extrema vertical superior. Presentaba una leve dismetría apendicular y ataxia de la marcha. No existía fatigabilidad. El 
resto de pares craneales, fuerza y sensibilidad no tenían alteraciones. La exploración física general estaba dentro de la normalidad, y el fondo de ojo, sin alteraciones.

En la RM cerebral se objetivaron numerosas lesiones puntiformes, hipertintensas en secuencia $\mathrm{T}_{2}$, de localización córtico-subcortical de ambos hemisferios. También se identificaban estas hiperintensidades de señal en el mesencéfalo, hipotálamo, tálamos, ganglios de la base, protuberancia y ambos hemisferios cerebelosos. Tras la administración de contraste paramagnético, mostraban captación, con un patrón miliar (Figura, a y b). Existía un leve realce del III y V pares craneales de forma bilateral.

Se le realizó estudio complementario con análisis de sangre: hemoglobina, 11,3 g/dL; colesterol, 286 mg/dL; y colesterol LDL, 195 mg/dL. La velocidad de sedimentación globular era de $56 \mathrm{~mm} / \mathrm{h}$ y la lactato deshidrogenasa, de $256 \mathrm{U} / \mathrm{L}$. El resto del hemograma, coagulación y bioquímica, incluyendo perfil tiroideo y vitaminas, no tenían alteraciones. El estudio de autoinmunidad (anticuerpos antinucleares, anticitopasma, microsomales, antimitocondriales, antimúsculo liso y antitiroideos) resultó negativo. El complemento y los marcadores tumorales estaban dentro de la normalidad. El estudio microbiológico no tenía alteraciones relevantes (serología para virus herpes, rubéola, Toxoplasma, Brucella, virus de la inmunodeficiencia humana, virus de la hepatitis B, virus de la hepatitis C, virus de EpsteinBarr y sífilis), y el Mantoux fue negativo. El inmunofenotipo en la sangre periférica no presentó alteraciones patológicas. El análisis del líquido cefalorraquídeo fue: aspecto claro; hematíes, $5 / \mathrm{mm}^{3}$; leucocitos, 13/ $\mathrm{mm}^{3}$ (el 100\% linfocitos); glucosa, 61 $\mathrm{mg} / \mathrm{dL}$; proteínas, $58 \mathrm{mg} / \mathrm{dL}$; y adenosina deaminasa, 4,8. El estudio microbiológico, bandas oligoclonales e inmunofenotipo fueron normales. La enzima conversora de angiotensina y los anticuerpos antineuronales en sangre y líquido cefalorraquídeo también fueron normales. La punción-aspiración con aguja fina de la adenopatía retrofaríngea hallada en las pruebas de neuroimagen no presentó datos que sugirieran malignidad.

Se realizó una arteriografía cerebral, que descartó anomalías vasculares, y se completó el estudio de neuroimagen con una RM medular, que fue normal.

Se llevó a cabo un estudio neurofisiológico con estimulación repetitiva de fibra única, que no mostraba alteraciones.

En la ecografía tiroidea se detectó un nódulo anecoico sugestivo de quiste, sin otros hallazgos. La ecografía mamaria no tenía alteraciones. La tomografía computarizada toracoabdominal presentó es-
Figura. Resonancia magnética craneal, antes ( $a$ y b) y después (c y d) del tratamiento con corticoides. Secuencias en $\mathrm{T}_{1}$ con contraste que muestran el patrón miliar de afectación tanto en el troncoencéfalo como en la región córtico-subcortical.

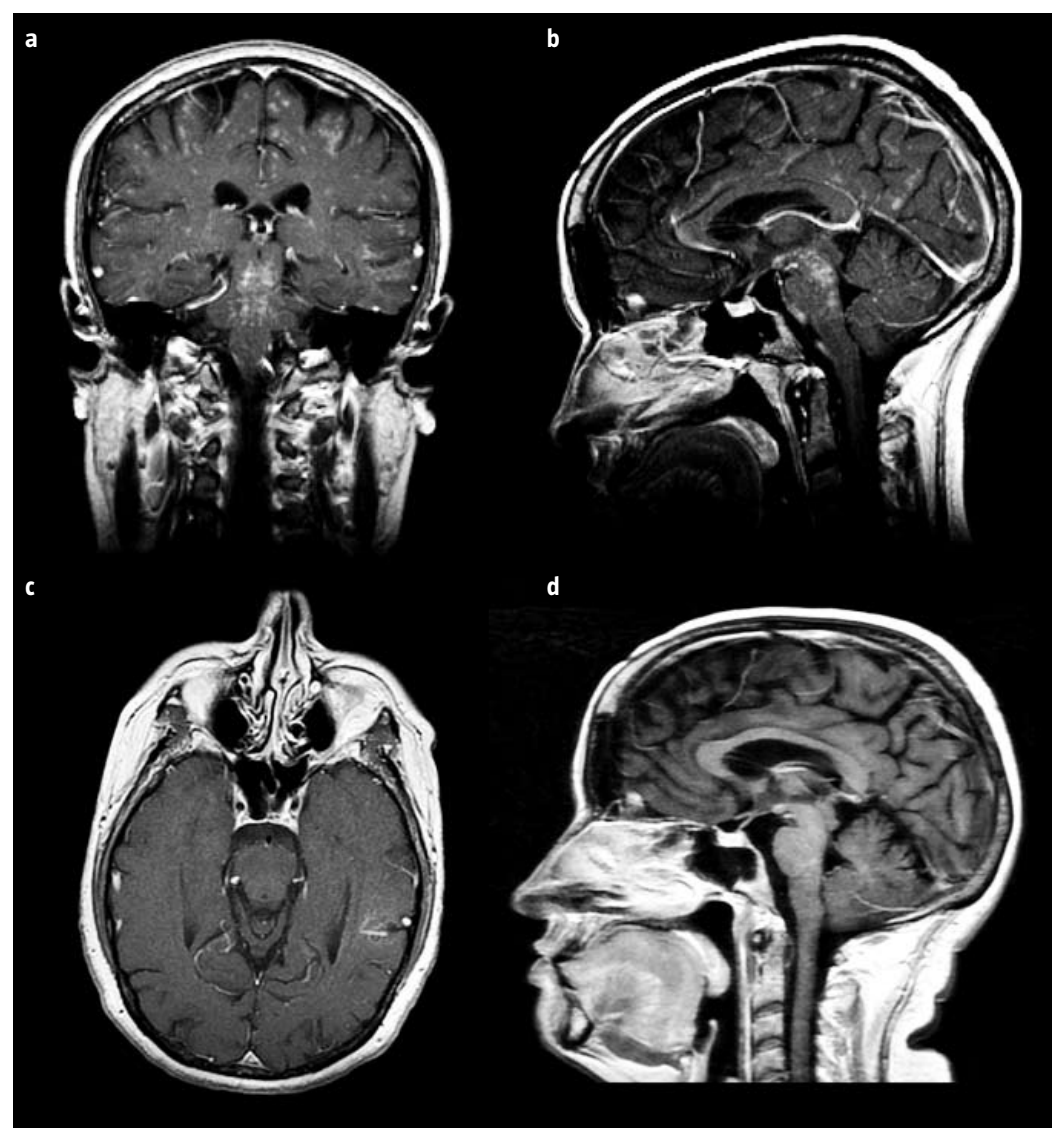

teatosis hepática sin otros hallazgos. La tomografía computarizada por emisión de positrones no mostró indicios de neoplasia subyacente.

La paciente presentó una remisión parcial espontánea en las siguientes semanas, por lo que se decidió no tratar inicialmente y realizar vigilancia clínica. Sin embargo, cuatro meses después experimentó empeoramiento, con un nuevo cuadro de disartria y ataxia. Se repitió la RM cerebral, donde persistían las hiperintensidades de señal puntiformes en número similar, pero con leve atenuación del realce poscontraste.

Ante la sospecha de origen inflamatorio del proceso, siendo negativo el estudio complementario realizado y los hallazgos en la RM cerebral compatibles con el síndrome CLIPPERS, se inició tratamiento con metilprednisolona intravenosa en dosis 
de $1 \mathrm{~g}$ /día durante cinco días, y descenso desde prednisona $1 \mathrm{mg} / \mathrm{kg}$ hasta dosis de mantenimiento de $20 \mathrm{mg} /$ día. La paciente mejoró hasta la práctica resolución de los síntomas.

En la tercera imagen por RM de control se visualizaba mejoría tanto en el número de lesiones como en la intensidad de la captación de contraste (Figura, c y d).

La paciente experimentó, sin embargo, un tercer episodio de ataxia y disartria pocos meses después, que requirió de nuevo metilprednisolona intravenosa durante cinco días y mantenimiento con $30 \mathrm{mg} /$ día de prednisona. Experimentó mejoría clínica hasta nueva resolución.

\section{Discusión}

Desde que Pittock et al [1] describiesen la entidad, se han publicado una veintena de casos más, ampliando el espectro clínico de este síndrome [2-9]. En ellos, las alteraciones oculomotoras y la ataxia están presentes en el diagnóstico prácticamente siempre. Además, se ha descrito paresia del V y VII pares craneales, nistagmo e incluso tetraparesia. En uno de estos trabajos se describe la presencia de alteraciones cognitivas a lo largo de la evolución en el contexto de pérdida axonal y atrofia en la neuroimagen de estos pacientes [10].

La característica radiológica principal es la aparición en la RM de lesiones captantes nodulares, puntiformes localizadas en puente con extensión variable hacia la médula, pedúnculos cerebelosos, cerebelo, ganglios basales y cuerpo calloso, pero con un gradiente de captación de las lesiones más débil conforme se alejan del puente [1]. En la serie de Simon et al [10] se cuestiona que la localización de las lesiones predomine siempre en el puente, se amplía este núcleo de captación predominante a los pedúnculos cerebelosos y el cerebelo, y se propone que el nombre del síndrome cambie a 'inflamación crónica linfocítica con realce perivascular pontocerebeloso'.

Se han descrito lesiones supratentoriales, pero suelen ser escasas y se localizan en la sustancia blanca (cuerpo calloso y tracto corticoespinal) y la sustancia gris (ganglios basales, hipocampo y núcleo dentado), y la localización cortical es muy rara. Se ha descrito la presencia de edema perilesional, captación anular del contraste e incluso RM inicial normal $[2,11]$.

La mayor parte de los casos publicados se apoya en la anatomía patología para el diagnóstico, que muestra una importante infiltración de linfocitos $\mathrm{T}$ de los espacios perivasculares del tronco del encéfalo, en su mayoría linfocitos T CD4 [1,2,4,6,8,10,11].

Sin embargo, no existe un biomarcador definido y la neuroimagen, aunque muy sugestiva, no es específica $[7,12-16]$. En el diagnóstico diferencial habría que considerar la neurosarcoidosis, el linfoma, el glioma, la histiocitosis, la esclerosis múltiple, la vasculitis aislada del sistema nervioso central, infecciones de sistema nervioso central, complicaciones neurológicas de enfermedades sistémicas autoinmunes y la encefalitis de Bickerstaff.

Existe controversia acerca de la necesidad de realizar biopsia cerebral para descartar otras enfermedades sistémicas y del sistema nervioso central: el hecho de que las lesiones en el síndrome CLIPPERS se localicen con frecuencia en zonas elocuentes, lo que conlleva un alto riesgo de complicaciones, junto con que la anatomía patológica no es específica, hace necesario plantearse su indicación con cautela, exceptuando aquellos casos de presentación atípica en los que no hay respuesta a los corticoides o si persiste la sospecha de un diagnóstico alternativo [1,13].

En nuestro caso, nos hemos basado en los hallazgos clínicos y de RM para realizar el diagnóstico de síndrome CLIPPERS. La paciente sufrió tres episodios de diplopía, disartria y ataxia que mejoraron espontáneamente en la primera ocasión y con corticoterapia en las siguientes. En la RM cerebral aparecieron las lesiones captantes características en el tronco del encéfalo, pero la distribución 'radial' típica no se respetaba y las lesiones aparecían igualmente concentradas en la región corticosubcortical de forma bilateral. Se objetivó, además, realce con gadolinio del III y V pares craneales, aunque sin evidencia de déficit de éstos en la exploración, hallazgos de RM no descritos hasta el momento en la bibliografía.

El curso clínico de nuestro caso sigue un patrón remitente-recurrente, sin evidencia hasta el momento de progresión de la enfermedad. En el estudio a cinco años, que incluye a 12 pacientes, de Taieb et al [11], se relaciona un tiempo más largo de remisión con la retirada más lenta de los corticoides, mientras que el desarrollo de secuelas lo hace con la gravedad de los brotes y con la presencia de atrofia en la neuroimagen.

La tendencia a la recurrencia en todos los pacientes, la evidencia de neurodegeneración en trabajos previos [10] y el fallecimiento de uno de los pacientes que no recibió tratamiento corticoideo les lleva a proponer que el tratamiento con corticoides con altas dosis debe ser precoz, con descenso lento y dosis de mantenimiento altas (> $20 \mathrm{mg} /$ día) a largo plazo. 
Los efectos adversos relacionados con el uso de corticoterapia crónica han motivado el ensayo con algunos inmunosupresores en regímenes variables (habitualmente en combinación con corticoterapia oral) en casos aislados.

Se tiene experiencia positiva con el uso de metotrexato en cuatro pacientes $[1,4,6,8]$, ciclofosfamida [6] en uno y rituximab en otro [11]. Los pacientes tratados con azatioprina, hidroxicloroquina, micofenolato e inmunoglobulinas intravenosas $[1,5,10]$ sufrieron recurrencias de la enfermedad durante el tratamiento.

La patogenia del síndrome CLIPPERS se desconoce. La presencia de un infiltrado linfocitario y la respuesta a inmunosupresores hacen que la etiología autoinmune sea la más aceptada. La localización de la inflamación en los espacios perivasculares sugiere la hipótesis de un proceso patológico inflamatorio primario venoso [1,11]. Algunos trabajos plantean un posible mecanismo inmunoalérgico [17] y en relación con la vacunación frente a la gripe [18].

Un aspecto no aclarado en la bibliografía es la razón por la que una entidad con tantas peculiaridades ha pasado desapercibida hasta la fecha. La posible existencia de formas benignas o autolimitadas de la enfermedad, junto con la mejoría técnica y la generalización de la RM, puede que hayan contribuido a su mejor identificación.

Serán necesarios estudios ulteriores para encontrar otros marcadores específicos que confirmen la etiopatogenia diferente que se sospecha. Por otro lado, parece necesario elaborar estrategias terapéuticas que establezcan la duración y beneficio a largo plazo de los corticoides, así como la idoneidad del inmunosupresor o inmunomodulador que, en combinación con los corticoides, permita la prevención a largo plazo de las recurrencias.

\section{Bibliografía}

1. Pittock SJ, Debruyne J, Krecke KN, Giannini C, Van den Ameele J, De Herdt V, et al. Chronic lymphocytic inflammation with pontine perivascular enhancement responsive to steroids (CLIPPERS). Brain 2010; 133: 2626-34.

2. Taieb G, Wacongne A, Renard D, Figarella-Branger D, Castelnovo G, Labauge P. A new case of chronic lymphocytic inflammation with pontine perivascular enhancement responsive to steroids with initial normal magnetic resonance imaging. Brain 2011; 134 (Pt 8): e182-3.
3. Duprez TP, Sindic CJ. Contrast-enhanced magnetic resonance imaging and perfusion-weighted imaging for monitoring features in severe CLIPPERS. Brain 2011; 134 (Pt 8): e184-6.

4. List J, Lesemann A, Wiener E, et al. A new case of chronic lymphocytic inflammation with pontine perivascular enhancement responsive to steroids. Brain 2011; 134 (Pt 8): e185-6.

5. Gabilondo I, Saiz A, Graus F, Villoslada P. Response to immunotherapy in CLIPPERS syndrome. J Neurol 2011; 258: 2090-2.

6. Kastrup O, Van de Nes J, Gasser T, Keyvani K. Three cases of CLIPPERS: a serial clinical, laboratory and MRI follow-up study. J Neurol 2011; 258: 2140-6.

7. Jones JL, Dean AF, Antoun N, Scoffings DJ, Burnet NG, Coles AJ. 'Radiologically compatible CLIPPERS' may conceal a number of pathologies. Brain 2011; 134 (Pt 8): e187.

8. Sempere AP, Mola S, Martín-Medina P, Bernabeu A, Khabbaz E, López-Celada S. Response to immunotherapy in CLIPPERS: clinical, MRI, and MRS follow-up. J Neuroimaging 2013; 23: 254-5.

9. Lefaucheur R, Bouwyn JP, Ahtoy P, Gérardin E, Derrey S, Maltête D. Teaching neuroimages: punctuate and curvilinear enhancement peppering the pons responsive to steroids. Neurology 2011; 77: e57-8.

10. Simon NG, Parratt JD, Barnett MH, Buckland ME, Gupta R, Hates MW, et al. Expanding the clinical, radiological and neuropathological phenotype of chronic lymphocytic inflammation with pontine perivascular enhancement responsive to steroids (CLIPPERS). J Neurol Neurosurg Psychiatry 2012; 83: 15-22.

11. Taieb G, Duflos C, Renard D, Audoin B, Kaphan E, Pelletier J, et al. Long-term outcomes of CLIPPERS (chronic lymphocytic inflammation with pontine perivascular enhancement responsive to steroids) in a consecutive series of 12 patients. Arch Neurol 2012; 69: 847-55.

12. Kira J. The expanding phenotype of CLIPPERS: is it a disease or a syndrome? J Neurol Neurosurg Psychiatry 2012; 83: 2-3.

13. Limousin N, Praline J, Motica O, Cottier JP, Rousselot-Denis C, Mokhtari K, et al. Brain biopsy is required in steroid-resistant patients with chronic lymphocytic inflammation with pontine perivascular enhancement responsive to steroids (CLIPPERS). J Neurooncol 2012; 107: 223-4.

14. Keegan BM, Pittock SJ. Cutting-edge questions about CLIPPERS (chronic lymphocytic inflammation with pontine perivascular enhancement responsive to steroids). Arch Neurol 2012; 69: 819-20.

15. Ortega MR, Usmani N, Parra-Herrán C, Adams DJ, Steingo B, Rammohan KW. CLIPPERS complicating multiple sclerosis causing concerns of CNS lymphoma. Neurology 2012; 79: 715-6.

16. Buttmann M, Metz I, Brecht I, Brück W, Warmuth-Metz M. Atypical chronic lymphocytic inflammation with pontocerebellar perivascular enhancement responsive to steroids (CLIPPERS), primary angiitis of the CNS mimicking CLIPPERS or overlap syndrome? A case report. J Neurol Sci 2013; 324: 183-6.

17. Tohge R, Nagao M, Yagishita A, Matsubara S. A case of chronic lymphocytic inflammation with pontine perivascular enhancement responsive to steroids (CLIPPERS) in East Asia. Intern Med 2012; 51: 1115-9.

18. Hillesheim PB, Parker JR, Parker JC Jr, Escott E, Berger JR. Chronic lymphocytic inflammation with pontine perivascular enhancement responsive to steroids following influenza vaccination. Arch Pathol Lab Med 2012; 136: 681-5. 


\section{CLIPPERS syndrome with atypical distribution of lesions in magnetic resonance imaging of the brain}

Introduction. CLIPPERS syndrome (chronic lymphocytic inflammation with pontine perivascular enhancement responsive to steroids) is an inflammatory process of the central nervous system whose distinguishing features are the enhancing punctiform lesions in the brainstem that appear in the magnetic resonance images. Clinically, it is accompanied by dysarthria, ataxia and diplopia, and usually responds to treatment with corticoids. Pathologically, T lymphocytes appear infiltrated in the perivascular spaces of the brainstem.

Case report. We report the case of a 40-year-old woman with an initial subacute clinical picture of binocular diplopia, ataxia and dysarthria. The magnetic resonance brain scan revealed $T_{2}$ hyperintense punctiform lesions in the stem, cerebellum, diencephalons and cortico-subcortical areas of both hemispheres, which were enhanced with contrast. An aetiological study was performed to rule out any underlying infectious, neoplastic or inflammatory origin, the results being negative. The patient was treated on two occasions with methylprednisolone, with a gradual lowering of the dosage, the response being favourable.

Conclusions. Diplopia and ataxia, as in our case, are practically always present. The MR findings consist of punctiform enhancing lesions located in the pons extending towards the cerebellum, basal ganglia and corpus callosum, the enhancement gradient becoming lower as the distance increases rostrally away from the cortex, and caudally towards the spinal cord. In the case of our patient, this gradient is not respected, and the density found was similar to that of lesions at the supratentorial level. The differential diagnosis is wide-ranging and justifies an extensive diagnostic study with, in certain cases, a biopsy study of brain tissue. The disease courses in a relapsing-remitting pattern and the earlier steroid therapy is established and the more prolonged it is, the better the prognosis will be.

Key words. CLIPPERS. Immunosuppressants. Magnetic resonance imaging. Neuroimmunology. Perivascular inflammation. Steroid therapy. 